\title{
Pleural complications of hydatid disease
}

\author{
Olgun Kadir Aribas, MDa \\ Fikret Kanat, $M D^{b}$ \\ Niyazi Gormus, MDc \\ Emel Turk, MD ${ }^{\mathrm{d}}$
}

From the Departments of Thoracic Surgery, ${ }^{\text {a }}$ Chest Diseases, ${ }^{\mathrm{b}}$ Cardiovascular Surgery, ${ }^{\mathrm{c}}$ and Infectious Diseases, ${ }^{\mathrm{d}}$ Medical School of Selcuk University, Konya, Turkey

Received for publication June 11, 2001; revisions requested July 18, 2001; revisions received Aug 2, 2001; accepted for publication Aug 3, 2001.

Address for reprints: Olgun Kadir Aribas, MD, Selcuk Universitesi Tip Fakultesi Hastanesi, Gogus Cerrahisi Anabilim Dali, 42080 Meram-Konya, Turkey (E-mail: olgun@selcuk.edu.tr).

J Thorac Cardiovasc Surg 2002;123:492-7

Copyright (C) 2002 by The American Association for Thoracic Surgery

0022-5223/2002 $\$ 35.00+0 \quad \mathbf{1 2 / 1 / 1 1 9 3 4 1}$

doi: $10.1067 / \mathrm{mtc} .2002 .119341$
Objective: The aim of this retrospective study was to review pleural and pericardial complications of patients with hydatid cysts and to analyze the management of therapy for these patients.

Methods: Between 1991 and 2001, 43 (29.7\%) of 145 patients presented with pleural and pericardial complications. These patients had spontaneous pneumothorax $(6.2 \%)$, empyema $(7.6 \%)$, pleural thickening (10.3\%), hepatopleural fistula $(2.8 \%)$, pericarditis $(2.1 \%)$, and hepatobronchial fistula $(0.7 \%)$. There were 22 male and 21 female patients, with a mean age of 30 years.

Results: The most common symptom was chest pain (79.1\%). In 37 (86.1\%) of 43 patients, the cysts were unilateral. The ratio of ruptured cysts was $88.4 \%$. In most of the patients, hydatid cysts developed in the right lung (62.9\%) and the lower lobes of the lung (70.4\%). Multiple cysts were found in $8(18.6 \%)$ patients. The most common surgical techniques were cystotomy with capitonnage $(55.7 \%)$ and decortication (69.8\%). Radical lung resection was used in $14 \%$ of the patients. The morbidity rate was $16.3 \%$, and the mortality rate was $2.3 \%$. The mean follow-up was 19 months with no recurrence.

Conclusions: Although lung-preserving surgical interventions should be preferred, radical surgical procedures have been used more commonly in patients with pleural complications of hydatid cysts, and the postoperative morbidity rate was higher in these patients. Because of this, the surgical treatment should be carried out before the development of pleural complications. In addition, echinococcosis should be considered and included in the differential diagnosis of spontaneous pneumothorax and empyema.

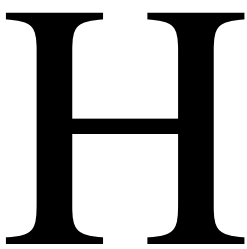
ydatid disease still remains an important health problem in Turkey. It is endemic in many sheep- and cattle-raising regions. The incidence of hydatid disease is 1:2000 in Turkey. ${ }^{1}$ Although it can locate in any place throughout the body, it is more common in the liver (75\%) and lung (25\%). ${ }^{2,3}$ However, in children it is most frequently encountered in the lung. ${ }^{1}$ Intrathoracic but extrapulmonary locations, which have been reported in $7.4 \%$ of patients, are very unusual. ${ }^{4}$ The most frequent complication of pulmonary hydatid disease is the rupture of the cyst into a bronchus, which is also regarded as a complicated cyst. ${ }^{2}$ If a cyst ruptures into the pleural or pericardial cavity, severe and life-threatening problems can be encountered. ${ }^{4-6}$

We present 43 cases of hydatid disease with pleural and pericardial complications and share our experiences by discussing the diagnostic and therapeutic procedures. 


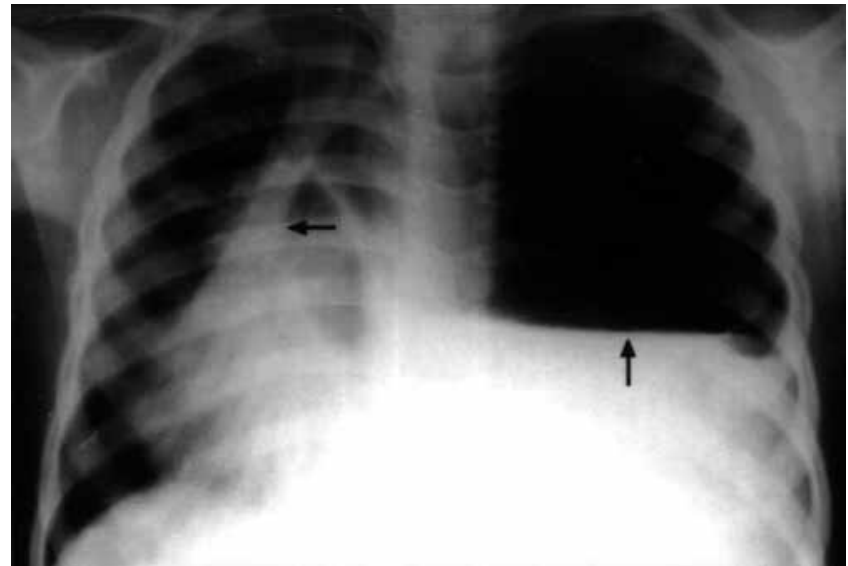

Figure 1. Chest radiograph of a patient with hydatid cyst presenting with tension pneumothorax.

\section{Materials and Methods}

The records of 145 patients with hydatid disease who had been hospitalized in the Department of Thoracic Surgery of Selcuk University School of Medicine between 1991 and 2001 were reviewed retrospectively. Among 145 patients, 43 (29.7\%) had pleural and pericardial complications of hydatid disease. Pleural complications were present in $40(27.6 \%)$ patients, and pericardial complications were seen in $3(2.1 \%)$ patients. Pleural complications were in the form of spontaneous pneumothoraxhydropneumothorax-tension pneumothorax (Figure 1) in $6.2 \%$, empyema-loculated empyema (Figure 2) in 7.6\%, severe pleural thickening in $10.3 \%$, and hepatopleural fistula (2.8\%), hepatobronchial fistula $(0.7 \%)$, or both in $3.5 \%$ of all patients with hydatid disease.

We evaluated the complications according to age; sex; occupation; symptoms; time period between detection of initial symptoms and hospitalization; radiologic and laboratory findings; distribution to the hemithorax and lobes; extrapulmonary localizations; site, shape, diameter, and number of cysts; means and types of surgical interventions; length of postoperative period; and postoperative morbidity and mortality.

The patients have been divided into 3 groups of pneumothorax, empyema, and severe pleural thickening according to the complication types, and these groups are assessed statistically for age (children-adolescent vs adult), sex (male vs female), hemithorax (right vs left), number of cysts (single vs multiple), size of cysts $(<10 \mathrm{~cm} \mathrm{vs}>10 \mathrm{~cm})$, time of admittance, and number of days of the postoperative period. Statistical analysis was performed with the $\chi^{2}$ test and Kruskal-Wallis variance analysis.

There were 22 male (51.2\%) and 21 female (48.8\%) patients, ranging from 5 to 67 years (mean, $30 \pm 18.73$ years; Table 1$)$. The male/female ratio was 1.04:1.

The initial diagnosis was based on chest radiography, ultrasonography (thorax and abdomen), and computed tomography of the thorax, excluding 5 patients in all cases. Echocardiography was used additionally in 4 patients with mediastinal widening. The Casoni skin test, the Weinberg complement fixation test, and

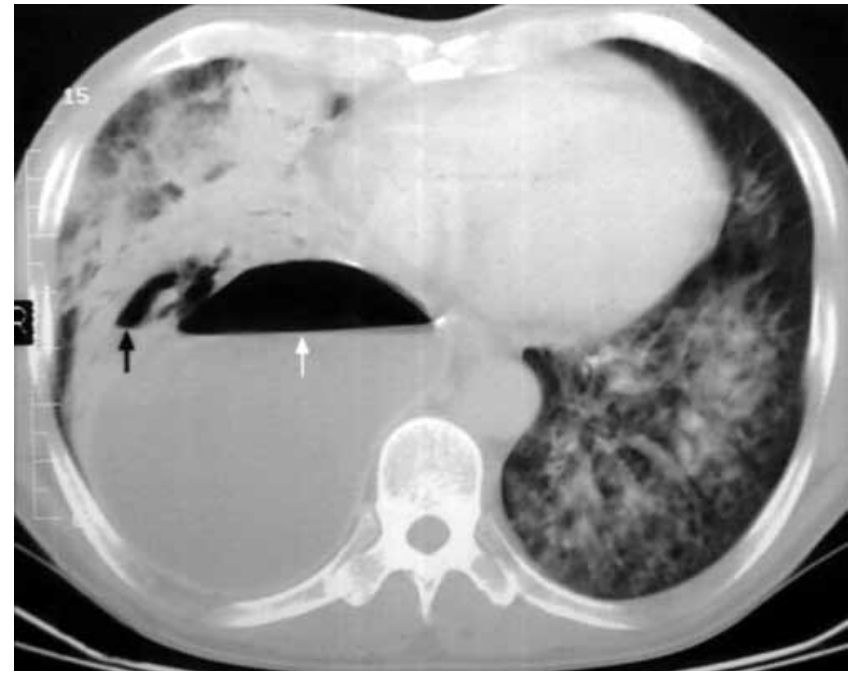

Figure 2. Chest computed tomographic scan of a patient with empyema revealing a cyst cavity in the posterobasal segment of the right lower lobe (black arrow) associated with a loculated pleural air-fluid level (white arrow).

eosinophilia measurement were also used in some cases. However, hemoglobin level, leukocyte count, and sedimentation rate were evaluated in all patients. Mean hemoglobin level $(11.85 \pm 1.83$ $\mathrm{mg} / \mathrm{dL}$; range, $7.70-15 \mathrm{mg} / \mathrm{dL}$ ) was in normal limits, whereas mean leukocyte count $(12,715.70 \pm 8011.46 / \mathrm{mL}$; range, $5560-52,660 / \mathrm{mL})$ and mean sedimentation rate $(42.83 \pm 30.06 \mathrm{~mm} / \mathrm{h}$; range, $6-108$ $\mathrm{mm} / \mathrm{h}$ ) were higher than normal. Pleural fluids collected from 11 patients with hydrothorax were cultured, and isolated organisms were Staphylococcus aureus in 3, Staphylococcus epidermidis in 2, Pseudomonas auriginosa in 2, and none in 4 patients.

All patients were treated surgically. In 13 patients admitted with pneumothorax and empyema, the closed-tube drainage procedure was applied initially, and they were operated on after the stabilization of their general status. However, the diagnosis of hydatid disease was made after 2 months in 2 patients. Posterolateral thoracotomy in $37(86 \%)$ patients, thoracophrenotomy in $4(9.3 \%)$ patients with liver-dome cysts, and sternotomy in $2(4.7 \%)$ patients with pericardial complications were performed. In patients with bilateral disease, staged thoracotomy (at least 2 months after the first) was preferred. Cystotomy with capitonnage or cystectomy with capitonnage were the most frequently used operation types. When the periphery of the cysts was destroyed or underwent bronchiectasis, segmentectomy or lobectomy was performed. In most of the cases, decortication was added to the procedures. A 3-cm Dacron patch was covered over the ascending aorta in a patient with pericardial complications, whereas the other pericardial defects were repaired with a patch. Thoracophrenotomy with cystectomy, subdiaphragmatic drainage, and thoracic decortication were carried out for the cysts in the liver dome, with abscess formation without pulmonary involvement. The germinative membrane from pulmonary cysts enucleated into the pleural cavity in 2 patients with pneumothorax and in 1 patient with empyema. In some broad-based cysts in which empyema 
TABLE 1. Distribution of mean age and number of patients according to sex and complications of hydatid disease

\begin{tabular}{|c|c|c|c|c|c|c|}
\hline \multirow[b]{2}{*}{ Sex } & \multicolumn{2}{|c|}{ Pleural complications } & \multicolumn{2}{|c|}{ Pericardial complications } & \multicolumn{2}{|c|}{ Total } \\
\hline & n (\%) & Mean \pm SD age & $\mathbf{n}(\%)$ & Mean \pm SD age & n (\%) & Mean $\pm S D$ age \\
\hline Male & $22(51.2)$ & $26.41 \pm 17.75$ & - & - & $22(51.2)$ & $26.41 \pm 17.75$ \\
\hline Female & $18(41.8)$ & $31.28 \pm 18.32$ & $3(7)$ & $49.00 \pm 18.32$ & $21(48.8)$ & $33.81 \pm 19.41$ \\
\hline Total & $40(93)$ & $28.60 \pm 17.94$ & $3(7)$ & $49.00 \pm 18.32$ & $43(100)$ & $30.02 \pm 18.73$ \\
\hline
\end{tabular}

TABLE 2. Data of symptoms ( $n=43)$

\begin{tabular}{lc}
\hline Symptoms & $\mathbf{n}(\%)$ \\
\hline Chest pain & $34(79.1)$ \\
Cough & $30(69.8)$ \\
Dyspnea & $19(44.2)$ \\
Sputum production & $16(37.2)$ \\
Fever & $13(30.2)$ \\
Hemoptysis & $6(13.9)$ \\
Spring-water expectoration & $8(18.6)$ \\
Vomiting & $2(4.7)$ \\
Abdominal pain & $2(4.7)$ \\
Membrane expectoration & $1(2.3)$ \\
Fatigue & $1(2.3)$ \\
Palpitation & $1(2.3)$ \\
Biloptysis & $1(2.3)$
\end{tabular}

developed, no capitonnage was performed. In the postoperative period, at least 2 months of mebendazole $\left(50 \mathrm{mg} \cdot \mathrm{kg}^{-1} \cdot \mathrm{d}^{-1}\right)$ or albendazole $\left(10 \mathrm{mg} \cdot \mathrm{kg}^{-1} \cdot \mathrm{d}^{-1}\right)$ therapy was given in all patients against a probable recurrence. The mean follow-up period was $19.44 \pm 10.18$ months (range, 4-40 months).

\section{Results}

Housewives $(37.2 \%)$, students $(23.3 \%)$, and self-employed persons $(16.3 \%)$ constituted the most frequent occupation groups. In all patients one or more symptoms were present. The most common symptom was chest pain $(79.1 \%$, Table 2). Only 2 patients had polycystic kidney and Lerich syndrome, and 1 patient had a history of trauma to the elbow. The mean period between the initial symptoms and admittance to the hospital was 96 days (range, 1-365 days). During that period, the patients were treated for pneumonia and bronchitis in other centers.

The most encountered pathologies detected in radiologic investigations were air-fluid level (23.3\%), homogeneous density $(23.3 \%)$, closure of costodiaphragmatic sinuses (20.9\%), and pulmonary air collapse (16.3\%). We also found mediastinal widening in $9.3 \%$, pneumonic infiltration in $7 \%$, water-lily sign with cavitary image in $4.7 \%$, and meniscus sign and elevation of the diaphragm in $2.3 \%$ of patients. With ultrasonographic examination, one or more hydatid cysts were established in the liver, mostly at the dome in 14 (32.6\%) patients. Ten (23.3\%) of 14 patients had cysts in both the liver and the lung. A subphrenic abscess in the cyst cavity was present in 4 (9.3\%) patients operated on previously. The mean diameter of 56 cysts detected in the lung and mediastinum was $6.12 \pm 3.22 \mathrm{~cm}$ (range, 2-15 cm), and the mean diameter of 17 cysts in the liver was $7.06 \pm 2.75 \mathrm{~cm}$ (range, $3-11 \mathrm{~cm}$ ). The diameter of the pulmonary cysts was less than $2 \mathrm{~cm}$ in $4.8 \%$ and more than $10 \mathrm{~cm}$ (giant cysts) in $7.1 \%$ and changed from 6 to 10 $\mathrm{cm}(38.1 \%)$ and 2 to $5 \mathrm{~cm}(50 \%)$ in most of the cases. The mean cyst diameters in children and adolescents less than 18 years old ( 15 patients) and in adults more than 18 years old (27 patients) were $5.00 \pm 2.2 \mathrm{~cm}$ (range, $2-10 \mathrm{~cm}$ ) and $5.43 \pm 2.62 \mathrm{~cm}$ (range, $2-15 \mathrm{~cm}$ ), respectively, and the difference between them was not significant $(P>.05)$. No significant difference was present between complication type and cyst size $(P>.05)$.

In our series $81.4 \%$ (35 patients) of the cysts were solitary (unilateral in 30 [69.8\%], bilateral in 3 [7\%], and mediastinal in $2[4.7 \%]$ ), and only $18.6 \%$ (8 patients) of the cysts were multiple (unilateral in 7 [16.3\%] and bilateral in 1 [2.3\%]). Overall, 54 cysts were resected from 37 (86\%) patients, of which $23(53.5 \%)$ had cysts in the right hemithorax and $10(23.3 \%)$ had cysts in the left hemithorax, and cysts were bilateral in $4(9.3 \%)$ patients. Otherwise, cysts were present only in the livers of $4(9.3 \%)$ patients and in the mediastinum of $2(4.7 \%)$ patients. The most frequent location of the hydatid cysts in our series was the right lung $(62.9 \%)$ and its lower lobes (70.4\%). Gathered results are demonstrated in Figure 3. All of the cysts (88.4\%) were suppurated, except for those in $5(11.6 \%)$ patients, and some were nonsuppurated and associated with suppurated multiple cysts in $16.3 \%$.

The most common procedure performed, as summarized in Table 3, was decortication (69.8\%). Radical pulmonary resection was carried out in $6(14 \%)$ patients with bronchiectasis or destroyed pulmonary areas. The mean postoperative period was $10.63 \pm 4.30$ days (range, $6-25$ days). No exitus was observed, but one (2.3\%) 60-year-old woman with a mediastinal hydatid cyst and pericardial complications died of multiple organ failure developing after postoperative onset of staphylococcus sepsis. 
TABLE 3. Surgical techniques used in the treatment of 43 patients with pleural and pericardial complications of hydatid cysts

\begin{tabular}{llc}
\hline Complication & Surgical techniques & $\mathbf{n ~ ( \% )}$ \\
\hline Pleural & Cystotomy + capitonnage & $5(11.5)$ \\
& Cystotomy + capitonnage + decortication & $19(44.2)$ \\
& Cystectomy + capitonnage + decortication & $7(16.3)$ \\
& Drainage + decortication & $3(7.0)$ \\
& Total (parenchyma-saving operations) & $34(79)$ \\
& Segmentectomy & $2(4.7)$ \\
& Lobectomy & $3(7.0)$ \\
& Lobectomy + decortication & $1(2.3)$ \\
Pericardial & Total (radical surgery) & $6(14.0)$ \\
& Cystectomy + pericardial patch & $2(4.7)$ \\
& Cystectomy + pericardial patch + & $1(2.3)$ \\
& transposition of ascending aorta & \\
Total & with Dacron graft & $3(7.0)$ \\
& Total (pericardial operations) & $43(100.0)$
\end{tabular}

However, postoperatively, 7 (16.3\%) patients had complications: $3(7.0 \%)$ had pneumonia, 2 (4.7\%) had atelectasis, 1 (2.3\%) had subdermal suppuration, and 1 (2.3\%) had insufficient lung expansion. No recurrence was observed in our patients followed up for a mean of $19.44 \pm 10.18$ months (range, 4-40 months).

\section{Discussion}

Hydatid disease is an infestation frequently caused by Echinococcus granulosus. It remains an important health problem, especially in regions with inadequate hygienic environments and veterinarian control. Therefore $90 \%$ of the patients with hydatid disease are from rural areas. ${ }^{2}$ The first station of the larvae taken orally through the portal system is the liver. Therefore it is more common in the liver (75\%), followed by the lungs. Although the larvae can enter the lungs through the lymphatic system or the bronchial system, it is thought that cysts settled down in the lungs are usually larvae that could have passed through the sinusoids. $1,2,7,8$ On the contrary to what is seen in adults, the lung is the most involved organ in children. ${ }^{1}$ Hydatid disease frequently involves only one organ, but cysts present both in lungs and liver comprise $10 \%$ of all cysts. ${ }^{2}$ However, in our series we found this ratio to be higher (32.5\%), with pleural complication. We found $18.5 \%$ and $14 \%$ of patients to have pneumothorax and empyema, respectively. The ratio of synchronous cysts located both in the lung and liver in children and adolescents in this study was less than that seen in adults, and it was $11.7 \%$ of all cases.

Hydatid disease is seen in any age and sex group, although it is more common in those 20 to 40 years of

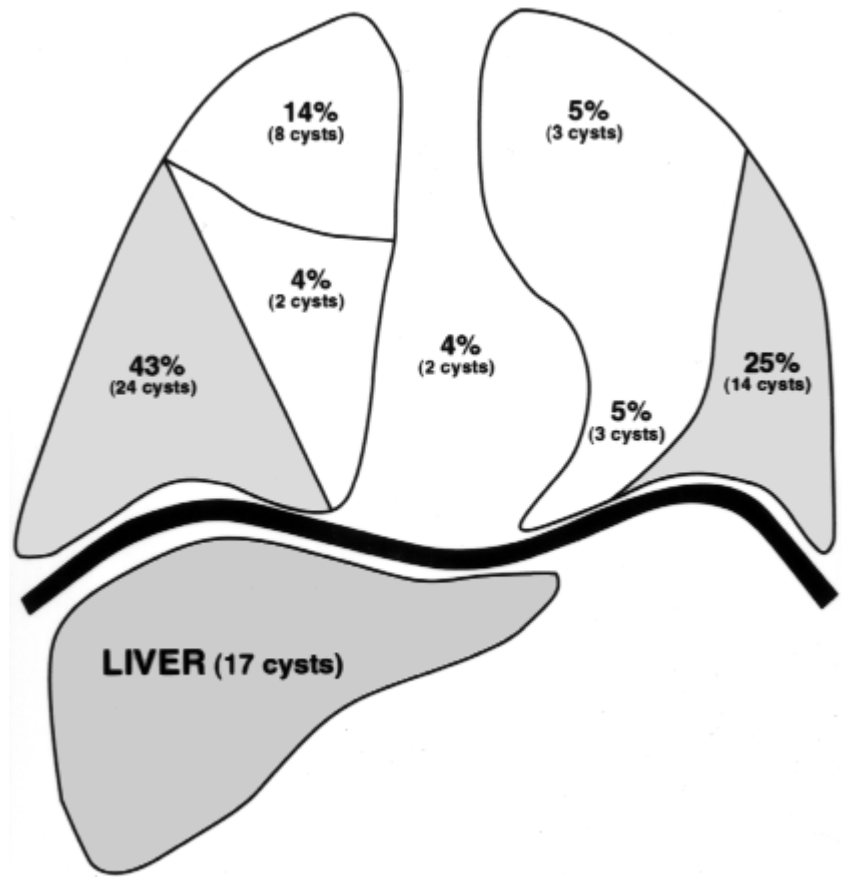

Figure 3. Distribution of the hydatid cysts with pleural and pericardial complications.

age. ${ }^{2,9}$ The results of our study were in harmony with those in the literature: the male/female ratio was 1.04:1, and the mean age of the patients was 30 years.

Pulmonary hydatid cysts locate in any pulmonary lobe but are more common in lower lobes, particularly those of the right lung. ${ }^{2,10}$ In our series cysts were located in the right lung $(61 \%)$ and in the lower lobes $(68 \%$; $43 \%$ in right lower lobe and $25 \%$ in left lower lobe).

In comparison with other organs, huge cysts are evident in the lungs. The elastic capability of the lungs without any resistance may give rise to the growth of cysts and, as in one of our patients, may occupy the whole hemithorax. Halezaroglu and colleagues ${ }^{11}$ noted that cysts larger than 10 $\mathrm{cm}$ would be seen in young persons because of the higher tissue elasticity. Yuksel and colleagues ${ }^{12}$ reported that the mean cyst diameter in patients less than 20 years old was $7.2 \mathrm{~cm}$, and intracystic pressure was $35.1 \mathrm{~cm} \mathrm{H}_{2} \mathrm{O}$. Cyst diameter in patients older than 20 years was measured significantly higher at $11.9 \mathrm{~cm}$; however, intracystic pressure of $38.1 \mathrm{~cm} \mathrm{H}_{2} \mathrm{O}$ in this age group was not found to be statistically significant. We did not find any significant mean cyst diameter between children and adolescents less than 18 years old (mean cyst diameter in 15 patients, $5.00 \pm 2.2 \mathrm{~cm}$ ) and adults more than 18 years old (mean cyst diameter in 27 patients, $5.43 \pm 2.62 \mathrm{~cm}, P>.05)$. Giant cysts, larger than $10 \mathrm{~cm}$, made up $7.1 \%$ and large cysts between 6 and $10 \mathrm{~cm}$ 
made up $38.1 \%$ of our series. In addition, we did not determine any difference between complication types and cyst sizes $(P>.05)$.

Although the Casoni skin test, the Weinberg fixation test, eosinophilia, and sedimentation rate are reported to be significant in ruptured cysts, ${ }^{8}$ they have not been routinely used recently because of low diagnostic efficiencies. ${ }^{2,7} \mathrm{We}$ did not study these tests routinely, except in a few cases. The most valuable diagnostic method in pulmonary hydatid disease is the plain chest radiograph. ${ }^{2,4,7,10,13}$ It ranges from typical images for intact cysts to appear as round or oval, sharp-edged homogeneous densities to images with meniscus sign, water-lilly sign, cavity with or without air-fluid levels, pneumonitis, or atelectasis of the lung for ruptured or complicated cysts. The most prominent radiologic finding of complicated cysts was the presence of an air-fluid level or only air between 2 layers of the cystic wall. ${ }^{11}$ Our patients with pleural complication of hydatid disease, except 5 of them, had ruptured cysts $(88.4 \%) ; 16.3 \%$ of patients with multiple cysts had both intact and ruptured cysts. In accordance with this, although only one fourth of the patients were determined to be homogeneous with welldefined opacity, three fourths of the patients had ruptured cyst appearances, most frequently with air-fluid level in our cases. Computed tomography of the chest clearly discriminates the cysts perforated and located behind or within the heart and the mediastinum. ${ }^{4,10} \mathrm{We}$ also found computed tomography of the chest to be of significant value in complicated hydatid cysts, especially with pleural thickening after an empyema and the infiltrative appearance of parenchyma, as well as in cysts that can not be noticed because of a small cavitary image developed after discharging its content into the pleural cavity. Ultrasonography and echocardiography are important diagnostic methods, particularly in the assessment of pericardial-cardiac and hepatic cysts. ${ }^{4}$ Hydatid cysts are encountered as intact forms, ruptured forms, or both in half of the patients. ${ }^{2}$ The hydatid cysts generally rupture into a bronchus, and these ruptured cysts are classified as complicated. The term complicated does not always indicate an infected or suppurated cyst. A perforated cyst without infection is also classified as complicated. ${ }^{11}$ The patients define this as a salty or peppery water expectoration, indicating spring-water expectoration, and it may even be an expectoration of membrane particles. ${ }^{2}$ We noticed a salty water expectoration in $18.6 \%$ of our patients and membrane expectoration in $2.3 \%$.

Although it is rarely seen, a more serious type of rupture is the perforation of the cyst into the pleural or pericardial cavity. 5,6,10,14 The increasing morbidity of these complications is the result of the rupture of cysts located in lungs or in the dome of the liver. ${ }^{2,3,5,6,8,10,14-16}$ Although pleural complications are reported to be $0.5 \%$ to $18.2 \%^{3,6,15,16}$ in the literature, we found a higher rate of these complications
(29.7\%). It is considered that the pleural necrosis after the pressure of pulmonary cysts, especially those located peripherally and subpleurally, has an important role in the rupture of cysts into the pleural cavity. ${ }^{3}$ In the cysts located at the dome of the liver, it is accepted that both the pressure and the irrigative effect of bile are responsible for the rupture of diaphragm. ${ }^{6}$ In this study hepatothoracic complications were seen in $3.5 \%$ of the patients, and the most important symptom in a patient with hepatobronchial fistula was biloptysis. The most important reason for the rarity of this complication is the dense adhesions between the parietal pleura and the cyst, inhibiting rupture into the pleural cavity. ${ }^{3}$ In our series severe pleural thickening was detected in $10.3 \%$ of 145 patients with hydatid disease. We found that $6.2 \%$ and $7.6 \%$ of the patients were admitted to our hospital with spontaneous pneumothorax and empyema, respectively.

The primary treatment of pulmonary hydatid disease is surgical intervention, ${ }^{1-16}$ although chemotherapy is used as a complement to operative treatment to avoid recurrences or performed in only inoperable cases because of insufficient cardiac performance status, pulmonary performance status, or both. ${ }^{2}$ The aim in surgical intervention is mainly the removal of the germinative membrane without causing intraoperative contamination and prevention of an intrapulmonary residual cystic space. For this purpose, varying techniques, such as enucleation, pericystectomy, and simple cystotomy with or without capitonnage of the pericystic space, can be chosen in appropriate conditions during the operation. ${ }^{1,9,10,11,17}$ Decision-making criteria in selecting operative techniques are closely related to the experience of the surgeon with the disease. ${ }^{11}$ However, the presence of complications, the risk of dissemination, the size of the cyst, and the vital importance of the involved organ are the other most commonly accepted criteria. ${ }^{8}$ Cystotomy or cystectomy and capitonnage are commonly applied operative procedures. $^{2,8,11}$ Enucleation is not a preferred procedure because of the risk of rupture; instead, fine-needle aspiration of the content of the cyst may be recommended, especially to prevent spillage and to decrease the intracystic pressure, aiding removal of the cyst. ${ }^{8,11}$ We usually carried out cystotomy with capitonnage $(55.7 \%)$ and cystectomy with capitonnage $(23.3 \%)$. When the cystic cavity was being obliterated, large bronchial openings were closed first, and then the cavity was obliterated by means of purse-string sutures from within (capitonnage) in our patients. However, in addition to those procedures, the decortication ratio of our experience was higher because of the pleural complications of the patients $(69.8 \%)$. Radical pulmonary resections should be performed when the pulmonary parenchyma around the cyst is destroyed, could not expand, or both. . $^{74}$ We applied this procedure in $14 \%$ of the complicated cases. This ratio, which is 2 to 3 times higher than those in other series, may be explained by the late admittance of the 
patients to the hospital (mean, 3 months), thus increasing the irreversible injury of the lung caused by infections.

Recently, the most popular intervention in multiple bilateral pulmonary cysts with or without hepatic cysts is sternotomy with anterior bilateral thoracotomy, providing removal of all cysts in one session. ${ }^{8,18}$ However, such interventions should not be preferred for the risk of mediastinitis in complicated cases (especially empyema). ${ }^{10}$ We consider it suitable only for patients with pericardial complications. Instead, staged thoracotomy is a safer and more reliable procedure. We also performed staged thoracotomy in 4 patients with bilateral cysts, the complicated side first and the contralateral side after 2 months. It is more appropriate to carry out thoracophrenotomy, which was applied in 4 patients of our series, if cysts are present in both the right lung and the liver or if a hepatic cyst causes only a pleural complication without a pulmonary cyst. If a hepatic cyst causes only a hepatobronchial fistula without pleural complication and a pulmonary cyst, the solely abdominal approach to hepatic cyst and fistula can be adequate. ${ }^{6}$

In most of the series the mortality ratio has been reported to be $0 \%$ to $2 \% .^{2,6,7,16,19}$ In our complicated cyst series it was $2.3 \%$. Recurrence is reported in $1.5 \%$ to $2 \%$ of the patients who are followed up for pulmonary hydatid disease, with mostly ruptured cysts, for a long period of time. ${ }^{2,14,19}$ We had no recurrence in our series, including mostly ruptured and pleural complications, in approximately 19 months of follow-up period. This may be a result of the postoperative chemotherapy given for 2 months to the patients, which is also proposed in the literature for the prevention of secondary recurrences in patients with pulmonary hydatid cysts after spontaneous or iatrogenic rupture of cysts and spillage of contents during an operation. ${ }^{2,10}$

Postoperative morbidity, mostly seen in synchronous pulmonary and hepatic cysts, was reported to be $3.5 \% .^{2}$ The ratio in our series consisting of complicated hydatid disease was $16.3 \%$. This finding may suggest that the morbidity in hydatid cysts with pleural complications is higher than in uncomplicated cases.

In conclusion, the hydatid cysts with pleural complications, pericardial complications, or both are associated with higher morbidity and more radical resection surgery than the others. Therefore prompt surgical intervention should be carried out after the diagnosis of hydatid disease without delay and before the development of complications.
In the differential diagnosis of empyema, pneumothorax, or both, hydatidosis should be considered and ruled out, especially in patients from the regions endemic for hydatidosis, and if so, surgical treatment is indicated as a primary therapy.

\section{References}

1. Mutaf O, Arikan A, Yazici M, Erdener A, Ozok G. Pulmonary hydatidosis in children. Eur J Pediatr Surg. 1994;4:70-73.

2. Dogan R, Yuksel M, Cetin G, Suzer K, Alp M, Kaya S, et al. Surgical treatment of hydatid cysts of the lung: report on 1055 patients. Thorax. 1989;44:192-9.

3. Ozer Z, Cetin M, Kahraman C. Pleural involvement by hydatid cysts of the lung. Thorac Cardiovasc Surg. 1985;33:103-5.

4. Oguzkaya F, Akcali Y, Kahraman C, Emirogullari N, Bilgin M, Sahin A. Usually located hydatid cysts: intrathoracic but extrapulmonary. Ann Thorac Surg. 1997;64:334-7.

5. Hadley M. Occult hydatid disease presenting as a spontaneous pneumothorax. Br J Radiol. 1985;58:770-2.

6. Kilani T, El Hammani S, Horchani H, Ben Miled-Mrad K, Hantous S, Mestiri I, et al. Hydatid disease of the liver with thoracic involvement. World J Surg. 2001;25:40-5.

7. Aytac A, Yurdakul Y, Ikizler C, Olga R, Saylam A. Pulmonary hydatid disease: report of 100 patients. Ann Thorac Surg. 1977;23:145-51.

8. Burgos R, Varela A, Castedo E, Roda J, Montero CG, Serrano S, et al. Pulmonary hydatidosis: surgical treatment and follow-up 240 cases. Eur J Cardiothorac Surg. 1999;16:628-35.

9. Zapatero J, Madrigal L, Lago J, Baschwitz B, Perez E, Candelas J. Surgical treatment of thoracic hydatidosis. A review of 100 cases. Eur J Cardiothorac Surg. 1989;3:436-40.

10. Topcu S, Kurul IC, Tastepe I, Bozkurt D, Gulhan E, Cetin G. Surgical treatment of pulmonary hydatid cysts in children. $J$ Thorac Cardiovasc Surg. 2000;120:1097-101.

11. Halezaroglu S, Celik M, Uysal A, Senol C, Keles M, Arman B. Giant hydatid cysts of the lung. J Thorac Cardiovasc Surg. 1997;113:712-7.

12. Yuksel M, Kir A, Ercan S, Batirel HF, Baysungur V. Correlation between sizes and intracystic pressures of hydatid cysts. Eur J Cardiothorac Surg. 1997;12:903-6.

13. Tuncer R, Orhan S, Sen N, Arslan R, Aydogdu I, Okur H, et al. Pulmonary hydatid cysts in children. Ann Med Sci. 2000;9:59-62.

14. Gashi M, Beqiri SB, Guguli M, Recica X, Ahmedi E. Our experiences in surgical treatment of thoracic echinococcosis during the period 1977-1986. Eur J Cardiothorac Surg. 1988;2:425-9.

15. Solak H, Yeniterzi M, Yuksek T, Anil N, Goktogan T, Ceran S. The hydatid cyst of the lung in children and results of surgical treatment. Thorac Cardiovasc Surg. 1990;38:45-7.

16. Athanassiadi K, Kalavrouziotis G, Loutsidis A, Bellenis I, Exarchos N. Surgical treatment of echinococcosis by a transthoracic approach: a review of 85 cases. Eur J Cardiothorac Surg. 1998;14:134-40.

17. Solak H, Ozgen G, Yuksek T, Eren N, Solak N, Akkoc O, et al. Surgery in hydatid cyst of the lung-a report of 460 cases. Scand J Thorac Cardiovasc Surg. 1988;22:101-04.

18. Rajinder S, Dhaliwal MCh, Maninder S, Kalkat MS. One-stage surgical procedure for bilateral lung and liver hydatid cysts. Ann Thorac Surg. 1997;64:338-41.

19. Qian ZX. Thoracic hydatid cysts: a report of 842 cases treated over a thirty-year period. Ann Thorac Surg. 1988;46:342-6. 